\title{
Human toxocariasis seroprevalence among patients with uveitis in Alborz Province, Iran
}

\author{
Mohammad Zibaei ${ }^{1,2, A-F \oplus}$, Maryam Alemi ${ }^{1, B \oplus}$, Natalia Marina Cardillo ${ }^{3, A, E \oplus}$, Hamid Derafshi ${ }^{1, A} \oplus$,

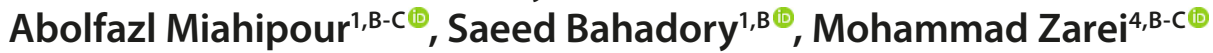 \\ ${ }^{1}$ Department of Parasitology and Mycology, School of Medicine, Alborz University of Medical Sciences, Karaj, Iran \\ ${ }^{2}$ Evidence-based Phytotherapy \& Complementary Medicine Research Center, Alborz University of Medical Sciences, Karaj, \\ Iran \\ ${ }^{3}$ National Scientific and Technical Research Council, Research Institute on Animal Production, Department of \\ Parasitology and Parasitic Diseases, Faculty of Veterinary Science, Buenos Aires, Argentina \\ ${ }^{4}$ Eye Research Center, Farabi Eye Hospital, Tehran University of Medical Sciences, Tehran, Iran \\ A - Research concept and design, B - Collection and/or assembly of data, C - Data analysis and interpretation, \\ $D$ - Writing the article, E - Critical revision of the article, F - Final approval of article
}

Zibaei M, Alemi M, Marina Cardillo N, Derafshi H, Miahipour A, Bahadory S, Zarei M. Human toxocariasis seroprevalence among patients with uveitis in Alborz Province, Iran. Ann Agric Environ Med. 2019; 26(1): 154-158. doi: 10.26444/aaem/102293

\begin{abstract}
Introduction and objective. Toxocariasis, predominantly caused by Toxocara canis, is a common zoonotic parasitosis worldwide. Toxocara infection is a cause of vision impairment and blindness. The presented study investigates the frequency of antibodies against Toxocara among uveitis patients and the epidemiological factors associated with disease.

Materials and method. Fifty-four patients with uveitis and 59 healthy subjects were studied. Anti-Toxocara antibodies status was determined in all serum samples using enzyme linked immunosorbent assay (ELISA), and seropositive samples analyzed by Western blot (WB) technique.

Results. The frequency of Toxocara canis infection was found to be significantly higher in uveitis patients, compared to healthy controls by the use of ELISA test, being $14.8 \%$ and $1.7 \%$, respectively. From 8 seropositive samples, 5 (62.5\%) patients exhibited Toxocara immunoglobulin G ( $\mathrm{ggG}$ ) antibodies in response to Western blot, whereas in the control group, none were detected positive by Western blot. No significant difference was found between pet owners, nor between different places of residence. The seroprevalence to Toxocara among uveitis patients was significantly related to gender, age and medical diagnosis. The highest prevalence was found in patients with posterior uveitis (27.8\%).

Conclusion. Anti-Toxocara antibody titers are associated with the risk of vision impairment -uveitis. The risk factor associated with Toxocara exposure identified in this study warrants further investigation.
\end{abstract}

\section{Key words}

toxocariasis, diagnosis, uveitis patients, Toxocara canis, ELISA, Western blot

\section{INTRODUCTION}

Toxocariasis is a worldwide zoonotic parasitic disease caused by the larval stage of either Toxocara canis (dog ascarid) or Toxocara cati (cat ascarid) [1]. Humans become infected with Toxocara species when they unintentionally ingest embryonated eggs that have been shed in the faeces of infected animals, or larvae through undercooked paratenic hosts [2, 3]. After ingestion of the eggs, infective larvae are released in the small intestine, and these subsequently penetrate the intestinal wall, enter the circulation, and migrate to different organs: the liver, lungs, brain, and eyes. where they induce inflammatory reactions and symptoms [4]. Based on the infected organs, it is clinically classified into visceral larva migrans (VLM), ocular larva migrans (OLM), neurotoxocariasis (NT), and covert toxocariasis (CT) $[5,6]$.

Toxocara larvae that migrate to the eye are trapped, resulting in an eosinophilic inflammatory mass. The syndrome takes several weeks to become clinically apparent, but the patients present with unilateral visual deficits, ocular pain, leukocoria, or strabismus. The retinal lesion

Address for correspondence: Mohammad Zibaei, Alborz University of Medical Sciences

e-mail: zibaeim@sums.ac.ir

Received: 15.07.2018; accepted: 22.12.2018; first published: 16.01.2019 most commonly presents with chronic unilateral uveitis, and a marked vitreous opacification that overlies a primary eosinophilic granuloma $[6,7]$. Posterior and peripheral retinochoroiditis, as well as optic papillitis, have also been reported. The inflammatory response created by ocular involvement may result in epiretinal membrane formation and traction retinal detachment [8]. Ocular toxocariasis is a clinically well-defined manifestation of intraocular infection by Toxocara larvae. The age at presentation in patients with ocular toxocariasis may vary from 1-77 years of age. Its clinical manifestation can be classified as posterior pole granuloma, peripheral granuloma, nematoda endophthalmitis, and atypical presentation $[9,10]$. In Japan, an epidemiologic study showed that ocular toxocariasis was recorded for $1.1 \%$ of all uveitis patients [11]. In a survey from the Republic of Korea, a high seroprevalence of $38.8 \%$ $(19 / 50)$ intermediate uveitis was reported by enzyme-linked immunosorbent assay in a Suwon population [12].

In Iran, Toxocara infection has been reported in most areas. The seroprevalence rate of human toxocariasis varies between $2.7-29.3 \%$. Reports from several regions also provide documented evidence of an increase in definitive hosts in recent years. The seroepidemiology of animal toxocariasis varies in different geographical regions, ranging from 3.3 to higher than $78.8 \%$ in different areas [13]. In a study in 
Karaj, an urban area in Iran, the prevalence of Toxocara spp eggs was $36.4 \%$ in public parks [14]. Therefore, the current study aimed to assess the seroprevalence of toxocariasis in ocular manifestation and the epidemiological factors associated with the disease in patients who are considered to have uveitis.

\section{MATERIALS AND METHOD}

Ethical clearance. The purpose and procedures of the study were explained to all participants, and a written informed consent was obtained from all of them. The study was approved by the ethical clearance committees of the Alborz University of Medical Sciences (No. ABZUMS. REC.1395, 103).

Study area. Karaj, in the centre of Alborz Province, is located in the north of Iran, bordering with the states of Tehran, Qazvin, Markazi, and Mazandaran. In 2011, the estimated population of Karaj was 2,412,513, of which $90.5 \%$ lived in urban areas. The study area ( $\left.35^{\circ} 48^{\prime} 57^{\prime \prime} \mathrm{N}, 50^{\circ} 58^{\prime} 29^{\prime \prime} \mathrm{E}\right)$ comprised the largest city in Alborz Province. The areas Karaj city and Alborz Province - are situated within a cold, semi-arid climatic zone, and receives most rainfall (annually $260 \mathrm{~mm}$ ) between March - May; mean annual temperature - $15.1^{\circ} \mathrm{C}$ (https://en.wikipedia.org/wiki/Alborz_Province).

Subjects. This study was conducted on 54 uveitis patients (21 women and 33 men) of the 272 individuals with intraocular inflammation of unknown etiology (various combinations of blurred or reduced vision, visual floaters, eye discomfort or pain, and intolerance to light), who were evaluated regarding their medical history, clinical characteristic at the Division of Ophthalmology, Educational and Therapeutic Centres or hospitals related to Alborz University of Medical sciences from April - December 2016. Inclusion criteria were inflammation of the uveal tract (iris, ciliary body, and choroid) diagnosed as anterior, intermediate, posterior uveitis, and idiopathic uveitis. Demographic characteristics of participants included age, gender, place of residence, keeping pets, history of diseases, and eye involvements, recorded during sample collection.

Control population. The control group consisted of 24 male and 35 female volunteers aged between 7-69 years with comparable epidemiology characteristics and without any complains or history of previous ocular complications in either themselves or their families. The demographic and lifestyle characteristics were obtained by study questionnaire.

Sample collection. Peripheral venous blood samples were collected during the same visit in which the patients and healthy volunteers were clinically examined to perform a haemogram with leukocyte differentiation, and measurement the red blood cells (RBCs) count. Eosinophilia was considered as an absolute eosinophil count of more than $350 / \mathrm{mm}^{3}$. Sera from participants were kept frozen at $-20^{\circ} \mathrm{C}$ until serological analysis.

Light microscopy. The blood smears were air-dried, fixed in methanol, stained with $3 \%$ Giemsa solution, and diluted in phosphate buffer saline ( $\mathrm{pH}=7.2)$ for $20 \mathrm{~min}$. The blood films were examined under a microscope with an oil immersion lens at a $1,000 \times$ power field. Haematological data, such as eosinophil levels, were recorded.

Toxocara-ELISA screening test. Anti-Toxocara canis antibodies (IgG) were detected by commercial EnzymeLinked Immunosorbent Assay (ELISA) kit (IBL, International Gmbh, Hamburg, Germany), following the manufacturer's guidance. In brief, diluted sera samples (1:100) were added to wells; after 30 min incubation at room temperature, horseradish peroxidase-conjugated goat antihuman IgG was added at a 1:1000 dilution (30 min at room temperature), followed by the tetramethylbenzidine (TMB) substrate. Absorbance readings were made at $620 \mathrm{~nm}$; cutoff absorbance value was defined as the mean absorbance reading for 3 negative control sera plus 2 standard deviations. Compared to negative and positive controls, samples with absorbance $\leq 9$ were considered negative while absorbance $\geq 11$ was considered positive, between $9.1-10.9$ as equivocal. Antibodies levels were expressed as reactivity indices, which were calculated as the cut-off value; positive samples had reactivity indices of more than 0.150 .

Toxocara-Western Blot analysis. Western Blot (WB) of the positive samples was performed and results interpreted according to the manufacturer's instructions, using WB IgG commercial kit (LDBIO Diagnostic, Lyon, France). Briefly, the strips were incubated for $120 \mathrm{~min}$ at $37^{\circ} \mathrm{C}$, with sera at a dilution of 1:100. Before being washed at least 3 times in PBS containing $0.1 \%$ Tween 20 , the strips were further incubated for $120 \mathrm{~min}$ at $37^{\circ} \mathrm{C}$ with the second antibody, anti-human IgG peroxidase conjugate (dilution 1:1,000). After washing, as mentioned previously, the substrate diaminobenzidine (DAB) was added and the reaction stopped by several washes in distilled water. The results were considered as positive when the samples reacted to 2 or more low-molecular-weight bands (LMWB $30-45 \mathrm{kDa}$ ).

Data analysis. Statistical evaluations were performed using SPSS software version 15.0. All epidemiological clinical and laboratory information were tested for association with Toxocara infection. Chi-squared and Fisher exact tests were used for categorical data. A $P$-value less than 0.05 was considered statistically significant.

\section{RESULTS}

IgG antibody positivity by ELISA with relation to demographic characteristics. Based on the evaluation by ELISA, of the 54 patients who participated in the study, $8 / 54(14.8 \%)$ had positive anti-Toxocara canis antibodies, compared to $1 / 59(1.7 \%)$ among the healthy group. The overall seroprevalence in the group of patients showed a significant dependence on gender and age $(P<0.05)-6.1 \%(2 / 33)$ in men and $28.6 \%(6 / 21)$ in women. The seroprevalence was highest $(28.6 \%, 4 / 14)$ in group $2(10-19$ years), followed by $14.3 \%(1 / 6)$ in group 5 ( $\geq 40$ years) (Tab. 1$)$. Uveitis patients who lived in rural areas demonstrated a higher seropositivity to Toxocara $(17.6 \%)$, compared to those in urban areas (13.5\%), but the difference did not attain a significance level $(P>0.05)$. Risk factors analysis indicated that the seropositive rates among the studied patients who kept pets or did not keep pets were $20.0 \%(4 / 20)$ and $11.8 \%(4 / 34)$, respectively $(P>0.05)$. 
Table 1. Seroprevalence to Toxocara canis determined by ELISA test in uveitis patients and control group with relation to demographical characteristics

\begin{tabular}{cccc}
\hline Factors & $\begin{array}{c}\text { Uveitis patients, } \\
\mathrm{N}(\%)\end{array}$ & $\begin{array}{c}\text { Control group, } \\
\mathrm{N}(\%)\end{array}$ & $\begin{array}{c}\text { Statistical analysis } \\
(P \text {-value }) \dagger\end{array}$ \\
\hline Age group (years) & & & \\
$0-9$ & $0 / 2(0.0)$ & $0 / 4(0.0)$ & $<0.05$ \\
$10-19$ & $4 / 14(28.6)$ & $0 / 14(0.0)$ & \\
$20-29$ & $0 / 4(0.0)$ & $1 / 5(20.0)$ & \\
$30-39$ & $3 / 27(11.1)$ & $0 / 30(0.0)$ & \\
$\geq 40$ & $1 / 7(14.3)$ & $0 / 6(0.0)$ &
\end{tabular}

Gender

$\begin{array}{llll}\text { Male } & 2 / 33(6.1) & 0 / 24(0.0) & <0.05 \\ \text { Female } & 6 / 21(28.6) & 1 / 35(2.9) & \end{array}$

Pet owner (cats and dogs)

$\begin{array}{llll}\text { Yes } & 4 / 20(20.0) & 0 / 15(0.0) & \text { Not significant } \\ \text { No } & 4 / 34(11.8) & 1 / 44(2.3)\end{array}$

Residency

$\begin{array}{llll}\text { Rural } & 3 / 17(17.6) & 0 / 20(0.0) & \text { Not significant } \\ \text { Urban } & 5 / 37(13.5) & 1 / 39(2.6)\end{array}$

Eosinophilia

Yes

$2 / 5(40.0)$

$1 / 11(9.1)$

$<0.05$

No

$6 / 49(12.2)$

$0 / 48(0.0)$

+ Significance by the $x^{2}$ test

Table 2. Seroprevalence of antibodies to Toxocara canis in the uveitis cases considering medical diagnosis

\begin{tabular}{lcc}
\hline Uveitis & Patients, N (\%) & Statistical analysis \\
\hline Anterior & $1 / 6(16.7)$ & $<0.05$ \\
Intermediate & $1 / 18(5.6)$ & \\
Posterior & $5 / 18(27.8)$ & \\
Idiopathic & $1 / 12(8.3)$ & \\
\hline
\end{tabular}

Regarding medical records, there was a predominance of posterior, uveitis compared to other forms of uveitis among the seropositive patients (Tab. 2). Of 8 seropositive patients, $27.8 \%(5 / 18)$ were associated with posterior, $16.7 \%(1 / 6)$ with anterior, $5.6 \%(1 / 18)$ with intermediate and $8.3 \%(1 / 12)$ with the idiopathic form of uveitis (Tab. 2). The dependence of the seropositivity to Toxocara canis on the clinical form of uveitis was statistically significant $(P<0.05)$.

IgG antibody positivity by ELISA with relation to microscopic blood analysis. Five of the 54 patients with uveitis (9.3\%) and 11 of the 59 healthy people (18.6\%) showed eosinophilia, with a range between $360-980 / \mathrm{mm}^{3}$. Among 5 patients with uveitis and eosinophilia, $2(40.0 \%)$ showed the presence of anti-Toxocara IgG antibodies, while among 11 healthy people with eosinophilia, there was only one person (9.1\%). The difference proved to be statistically significant $(P<0.05)$ (Tab. 1). This result suggests an association between the uveitis caused by Toxocara and eosinophilia.
Western blot. Of 8 seropositive samples by ELISA, 5 (62.5\%) tested positive for IgG antibodies against the excretorysecretory larval antigens of Toxocara canis by Western blot (TcES-WB). The positive WB strips showed at least 2 or more bands of lower molecular weights (Fig. 1).

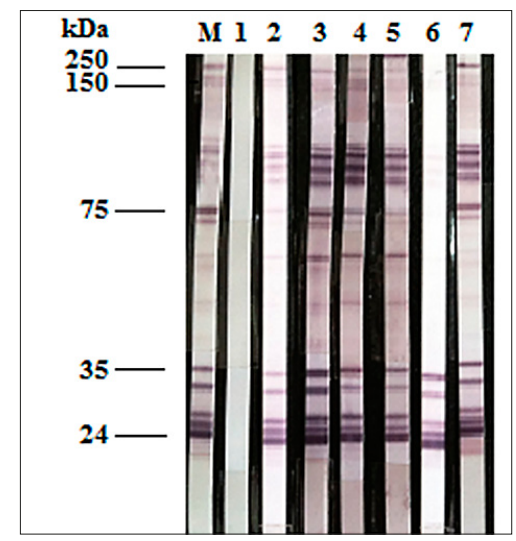

Figure 1. Western blot results for sera of the studied population whose IgG antibodies were reactive to low-molecularweight bands at 30 and $35 \mathrm{kDa}$, which are specifically related to Toxocara infection. Lane M: protein molecular weight marker; lane 1: toxocariasis-negative serum; lane 2: toxocariasis-positive serum; lanes 3-7: sera of patients with Toxocara canis (ELISApositive reacted).

\section{DISCUSSION}

Human infection by Toxocara spp. may result in visceral and ocular larva migrans with various types of morbidity and has thus drawn considerable attention in recent years [15]. Toxocariasis has a worldwide geographical distribution and the seropositivity of Toxocara antibodies varies from $2.4 \%$ $-76.6 \%$ [16-18]. Although human toxocariasis is one of the most common zoohelminthic infections worldwide, there are only a few studies that estimate the frequency of ocular larva migrans. The annual incidence of ocular toxocariasis in different parts of the word ranges from $17-52$ per 100,000 persons, and the prevalence ranges from $58-115$ per 100,000 people [9]. Ocular toxocariasis can induce inflammation of the uveal and retinal tissues, which has been revealed as uveitis, and it can result in diverse lesions, from a simple peripheral pigmentary retinal scar to macular damage that cause severe visual loss after treatment [9].

In this study, the frequency of Toxocara infection in uveitis patients was $14.8 \%$, significantly higher than in healthy controls (1.7\%). However, as the reported prevalence of toxocariasis in the general population in the area examined ranged from $2-25.6 \%$, the relatively low frequency of Toxocara infection in the control group required cautious interpretation. Using excretory-secretory antigens of Toxocara canis larvae by ELISA (TcES-ELISA), a same in the current study, association studies between toxocariasis and uveitis in a Korean population revealed anti-Toxocara antibodies (IgG) in $38.0 \%$ of the 50 participants [12]. Different serological studies have been undertaken regarding the seroprevalence of toxocariasis in patients with ocular inflammatory disorder in different geographical regions of the world $[19,20]$. The rate 
of seroprevalence of toxocariasis in the presented findings is very similar to the rate of infection in other parts of the word [21-24].

In most laboratories, the current methods of detecting T. canis infection in humans are typically based on TcESELISA [12]. Although TcES-ELISA has been reported on yield reasonable levels of specificity (96.7\%) and sensitivity (97.0\%), the specificity is excessively low to be reliable when assaying communities in which several of intestinal cross reacting helminths are common [19]. Thus Western blot, rather than a general ELISA, was employed in the current survey. Immunoblotting results of 8 ELISA seropositive samples in the patients and one case in healthy control in the present study showed that $62.5(5 / 8)$ was positive by TcES-WB. Limited studies are available in seroprevalence of toxocariasis in patients with uveitis. In an investigation, it has been shown that the seroprevalence of toxocariasis in uveitis patients using Toxocara excretory secretory-WB (TES-WB) was $2.6 \%$ [20].

Results of the presented study suggest a high (40.0\%) seroprevalence of Toxocara exposure in patients with eosinophilia. The seroprevalence found in these patients was higher than those $(9.1 \%)$ reported in the control group $(P<0.05)$. A similar study by Kwon et al. [12], investigated the association between intermediate uveitis and toxocariasis in a Korean population. In the analyses of intermediate uveitis patients with or without ocular toxocariasis, the increasing eosinophils was significantly different between the 2 groups. The seropositivity rate to Toxocara infection in the hypereosinophilia patients with intermediate uveitis were significantly higher than in non-ocular toxocariasis group [12].

According to the literatures, multiple risk factors have been associated with Toxocara epidemiology, such as age, gender, geophagia, keeping pets, playing in sand boxes, and climatic condition $[25,26]$.

In the presented study, seropositive results were obtained in females, a finding that could be explained by the culture of the area where women more than men come into contact with cats and dogs. The findings of the current study are in sharp contrast with those of Einipour et al. [20] and Kwon et al. [12], who reported that toxocariasis predominantly in males.

The major risk population in the study was the age group 10-19 years, significantly associated with seropositivity. Toxocariasis is seen frequently among children and young adults probably due to poor hygiene. The relatively gradual increase in seropositivity rate associated with age suggested that soil exposure, which is greatest during childhood years, may not be the principal mechanism by which people are exposed to Toxocara [27]. In the current study, uveitis patients consisted of various age groups, from children to the elderly, with ages ranging between $8-87$ years-old. The seropositivity was affected by age, though there were significantly more seropositive uveitis patients who were students. The current findings support a previous observation made by Pak et al. [25].

Contact with cats and dogs might represent a risk for toxocariasis. The presented results indicated that the subjects in both groups who exhibited a history of keeping dogs and cats demonstrated a higher risk of Toxocara infection than those who did not; however, these effects were non-significant in the statistical analysis. This finding could be explained by the culture of the area, where persons are less likely to come into contact with dogs and cats, or keep them in the home.

Another risk factor identified in the presented study was the rural residence, which could be explained by the diet of people in the region, who had consumed encapsulated larvae in the raw or uncooked meat of the paratenic hosts, such as cows, sheep, and chickens.

A main finding of the presented study was the demonstration of a significant relationship between seropositivity to Toxocara and the type of inflammation of the uvea. Of the 8 patients classified as having anti-Toxocara antibodies, 5 (27.8\% of total examined and $62.5 \%$ of total positive) had a posterior uveitis. This observation suggested that ocular toxocariasis can induce inflammation of uveal and retinal tissues that results in a posterior uveitis. These findings confirmed the earlier results indicating that posterior uveitis is the most common form of clinical presentation, consisting of $44.0 \%$ of ocular toxocariasis cases [28]. A case of ocular toxocariasis with posterior pole granuloma has also been reported [23].

\section{CONCLUSIONS}

Taken together, the obtained results corroborate some earlier observations on an association between Toxocara infection and uveitis. Thus, the investigation of anti-Toxocara IgG using ELISA should be a routine test in the people with retinal granuloma or uveitis, particularly in its posterior form.

\section{REFERENCES}

1. Despommier D. Toxocariasis: clinical aspects, epidemiology, medical ecology, and molecular aspects. Clinical Microbiol Rev. 2003; 16: 265-272.

2. Maraghi S, Jafari KM, Sadjjadi SM, Latifi SM, Zibaei M. Study on the contamination of Abadan public parks soil with Toxocara spp. eggs. J Environ Health Sci Eng. 2014; 12: 86.

3. Zibaei M, Sadjjadi SM, Maraghi S. The occurrence of Toxocara species in naturally infected broiler chickens revealed by molecular approaches. J Helminth. 2017; 91: 633-636.

4. Błaszkowska J, Góralska K, Wójcik A, Kurnatowski P, Szwabe K. Presence of Toxocara spp. eggs in children's recreation areas with varying degrees of access for animals. Ann Agric Environ Med. 2015; 22: $23-27$.

5. Borecka A, Kłapeć T. Epidemiology of human toxocariasis in Poland-A review of cases 1978-2009. Ann Agric Environ Med. 2015; 22: 28-31.

6. Brown GC, Tasman WS. Retinal arterial obstruction in association with presumed Toxocara canis neuroretinitis. Ann Ophthalmol. 1981; 13: $1385-1387$.

7. Jee D, Kim KS, Lee WK, Kim W, Jeon S. Clinical features of ocular toxocariasis in adult Korean patients. Ocul Immunol Inflamm. 2015; 24: $207-216$.

8. Sabrosa NA, de Souza EC. Nematode infections of the eye: toxocariasis and diffuse unilateral subacute neuroretinitis. Curr Opin Ophthalmol. 2001; 12: 450-454.

9. Ahn SJ, Ryoo NK, Woo SJ. Ocular toxocariasis: clinical features, diagnosis, treatment, and prevention. Asia Pac Allergy. 2014; 4: 134.

10. Zibaei M, Sadjjadi SM, Jahadi-Hosseini SH. Toxocara cati larvae in the eye of a child: a case report. Asian Pac J Trop Biomed. 2014; 4: S53-S55.

11. Goto H, Mochizuki M, Yamaki K, Kotake S, Usui M, Ohno S. Epidemiological survey of intraocular inflammation in Japan. Jap J Ophthalmol. 2007; 51: 41-44.

12. Kwon JW, Sim Y, Jee D. Association between intermediate uveitis and toxocariasis in the Korean population. Medicine. 2017; 96: e5829.

13. Zibaei M, Sadjjadi SM. Trend of toxocariasis in Iran: a review on human and animal dimensions. Iranian J Vet Res. 2017; 18: 233-242.

14. Zibaei M, Bahadory M, Cardillo N, Khatami AR. Soil Contamination with eggs of Toxocara species in public parks of Karaj, Iran. Int J Enteric Pathog. 2017; 5: 45-48. 
15. Sánchez TJ, López GJ, González NM, Villaseca DE, Manieu MD, Roizen BA, et al. Prevalence of ocular lesions in children seropositive to Toxocara canis. Revista Chilena Infecto. 2011; 28: 431-434.

16. Fan CK, Hung CC, Du WY, Liao CW, Su KE. Seroepidemiology of Toxocara canis infection among mountain aboriginal schoolchildren living in contaminated districts in eastern Taiwan. Trop Med Int Health. 2004; 9: 1312-1318.

17. Stensvold CR, Skov J, Moller LN, Jensen PM, Kapel CM, Petersen E, et al. Seroprevalence of human toxocariasis in Denmark. Clin Vaccine Immunol. 2009; 16: 1372-1373.

18. Fu CJ, Chuang TW, Lin HS, Wu CH, Liu YC, Langinlur MK, et al. Seroepidemiology of Toxocara canis infection among primary schoolchildren in the capital area of the Republic of the Marshall Islands. BMC Infect Dis. 2014; 14: 261.

19. Zibaei M, Sadjjadi SM, Sarkari B, Uga S. Evaluation of Toxocara cati excretory-secretory larval antigens in serodiagnosis of human toxocariasis. J Clin Lab Anal. 2015; 30: 248-253.

20. Einipour S, Jalusian F, Ramezani A, Abadi A, Hajialiani F, Zeinali S, et al. Toxocariasis antibody tittering in patients with uveitis. Koomesh. 2016; 17: 903-909.
21. Sharkey JA, McKay PS. Ocular toxocariasis in a patient with repeatedly negative ELISA titre to Toxocara canis. Br J Ophthalmol. 1993; 77: 253-254.

22. Altcheh J, Nallar M, Conca M, Biancardi M, Freilij H. Toxocariasis: clinical and laboratory features in 54 patients. An Pediatr (Barc). 2003; 58: 425-431.

23. Hashida N, Nakai K, Nishida K. Diagnostic evaluation of ocular toxocariasis using high-penetration optical coherence tomography. Case Rep Ophthalmol. 2014; 5: 16-21.

24. Pak KY, Park SW, Byon IS, Lee JE. Ocular toxocariasis presenting as bilateral scleritis with suspect retinal granuloma in the nerve fiber layer: a case report. BMC Infect Dis. 2016; 16: 426.

25. Good B, Holland CV, Taylor MRH, Larragy J, Moriarty P, Regan MO. Ocular toxocariasis in schoolchildren. Clin Infect Dis. 2004; 39 : 173-178.

26. Chávez JPB, Díaz RH, Peña AH, Isaías RC, Yrma EB, Gonzalez WR, et al. Human toxocariosis in Peru: Aspects of epidemiology, clinical and laboratory. Acta Med Peruana. 2011; 28: 228-236.

27. Yang HK, Woo SJ, Hwang JM. Toxocara optic neuropathy after ingestion of raw meat products. Optom Vis Sci. 2014; 91: e267-273.

28. Wilkinson CP, Welch RB. Intraocular Toxocara. Am J Ophthalmol. 1971; 71: 921-930. 